Bull. Korean Math. Soc. 42 (2005), No. 4, pp. 889-900

\title{
A COMPREHENSIVE STUDY OF SECOND ORDER MOCK THETA FUNCTIONS
}

\author{
BHASKaR SRIVASTAVA
}

\begin{abstract}
We consider the second order mock theta functions defined by McIntosh and define generalized functions. We give integral representation and multibasic expansion of these functions. We also show that they are $F_{q}$-functions.
\end{abstract}

\section{Introduction}

In his last letter to Hardy[9, pp. 354-355] four months before he died, Ramanujan wrote about functions whom he called 'mock' theta functions. He explained what he meant by mock theta function. It is a function $f(q)$ defined by a $q$-series which converges for $|q|<1$ and which satisfies the following two conditions:

(0) For every root of unity $\zeta$, there is a theta function $\theta_{\zeta}(q)$ such that the difference $f(q)-\theta_{\zeta}(q)$ is bounded as $q \rightarrow \zeta$ radially.

(1) There is no single theta function which works for all $\zeta$, i.e., for every theta function $\theta(q)$ there is some root of unity $\zeta$ for which $f(q)-\theta(q)$ is unbounded as $q \rightarrow \zeta$ radially.

He listed seventeen functions and classified them as of order three, five and seven. Later, in Ramanujan's "Lost" Notebook there were eleven identities involving seven functions and Andrews and Hickerson[3] called these seven functions mock theta functions of order six. Recently Choi[4] considered two identities found in the "Lost" Notebook of Ramanujan involving four functions and called them mock theta functions of order ten. Ramanujan did not say what he meant by the 'order' of the function.

Very recently Gordon and McIntosh $[6]$ in their brilliant paper gave a method of constructing mock theta function from ordinary theta series

Received November 25, 2004.

2000 Mathematics Subject Classification: 11B65, 33D15.

Key words and phrases: Mock theta functions, $q$-multibasic series. 
using half-shift transformation. They constructed eight functions and called them of order eight. They also gave a rigorous definition of the order of the function [6].

Recently McIntosh[7] has defined two functions and called them of order two. He has established relations between these mock theta functions and his eight order mock theta functions.

In this paper we make a comprehensive study of these second order mock theta functions. In section 4 we give Hecke type expansions for these second order mock theta functions. In a subsequent paper we will show that using these expansions these mock theta functions come out as coefficients of $z^{0}$ in certain power series expansion in $z$. In my paper [10], I have shown that Ramanujan's sixth order mock theta functions and Gordon and McIntosh's eighth order mock theta functions can be written as a coefficient of $z^{0}$ in the Laurent series expansion of rational functions of theta functions.

In Section 5, we define generalized functions which on specialization give these mock theta functions. We show that these generalized functions and consequently the mock theta functions are $F_{q}$-functions

In Section 6, we represent these generalized functions as an Integral.

In Section 7, we expand these generalized functions as a multibasic hypergeometric series and the same follows for these mock theta functions.

In Section 8, we represent these mock theta functions as continued fractions.

\section{Notation}

We shall use the following usual basic hypergeometric notations:

For $\left|q^{k}\right|<1$,

$$
\begin{aligned}
\left(a ; q^{k}\right)_{n} & =(1-a)\left(1-a q^{k}\right) \ldots\left(1-a q^{k(n-1)}\right), \quad n \geq 1 \\
\left(a ; q^{k}\right)_{0} & =1 \\
\left(a ; q^{k}\right)_{\infty} & =\Pi_{m=0}^{\infty}\left(1-a q^{m k}\right) .
\end{aligned}
$$

For convenience we shall write

$$
\left(a_{1}, a_{2}, \ldots, a_{k} ; q^{k}\right)_{n}=\left(a_{1} ; q^{k}\right)_{n}\left(a_{2} ; q^{k}\right)_{n} \ldots\left(a_{k} ; q^{k}\right)_{n}
$$

When $k=1$, we usually write $(a)_{n}$ and $(a)_{\infty}$ instead of $(a ; q)_{n}$ and $(a ; q)_{\infty}$, respectively. 
For non-negative integers $n$, we have

$$
\left(a ; q^{k}\right)_{n}=\frac{\left(a ; q^{k}\right)_{\infty}}{\left(a q^{n k} ; q^{k}\right)_{\infty}}
$$

and for other real $n$, we will take this as the definition of $\left(a ; q^{k}\right)_{n}$.

$$
\begin{aligned}
& \phi\left[\begin{array}{l}
\left.a_{1}, \ldots, a_{r}: c_{1,1}, \ldots, c_{1, r_{1}}: \ldots: c_{m, 1}, \ldots, c_{m, r_{m}} ; q, q_{1}, \ldots, q_{m} ; z\right] \\
b_{1}, \ldots, b_{s}: e_{1,1}, \ldots, e_{1, s_{1}}: \ldots: e_{m, 1}, \ldots, e_{m, s_{m}}
\end{array}\right] \\
& =\sum_{n=0}^{\infty} \frac{\left(a_{1}, \ldots, a_{r} ; q\right)_{n}}{\left(q, b_{1}, \ldots, b_{s} ; q\right)_{n}} z^{n}\left[(-1)^{n} q^{\frac{n^{2}-n}{2}}\right]^{1+s-r} \\
& \times \Pi_{j=1}^{m} \frac{\left(c_{j, 1}, \ldots, c_{j, r_{j}} ; q_{j}\right)_{n}}{\left(e_{j, 1}, \ldots, e_{j, s_{j}} ; q_{j}\right)_{n}}\left[(-1)^{n} q_{j}^{\frac{n^{2}-n}{2}}\right]^{s_{j}-r_{j}}
\end{aligned}
$$

For $x \neq 0$ and $|q|<1$,

$$
j(x, q)=(x, q / x, q ; q)_{\infty} .
$$

If $a$ and $m$ are integers with $m \geq 1$, then

$$
\begin{aligned}
& J_{a, m}=j\left(q^{a}, q^{m}\right), \\
& \bar{J}_{a, m}=j\left(-q^{a}, q^{m}\right), \\
& J_{m}=J_{m, 3 m}=\left(q^{m} ; q^{m}\right)_{\infty} .
\end{aligned}
$$

\section{Second order Mock theta functions}

The second order mock theta function defined by McIntosh[7] are

$$
\begin{aligned}
& A(q):=\sum_{n=0}^{\infty} \frac{q^{(n+1)^{2}}\left(-q ; q^{2}\right)_{n}}{\left(q ; q^{2}\right)_{n+1}^{2}}=\sum_{n=0}^{\infty} \frac{q^{n+1}\left(-q^{2} ; q^{2}\right)_{n}}{\left(q ; q^{2}\right)_{n+1}} \\
& B(q):=\sum_{n=0}^{\infty} \frac{q^{n^{2}+n}\left(-q^{2} ; q^{2}\right)_{n}}{\left(q ; q^{2}\right)_{n+1}^{2}}=\sum_{n=0}^{\infty} \frac{q^{n}\left(-q ; q^{2}\right)_{n}}{\left(q ; q^{2}\right)_{n+1}}
\end{aligned}
$$

and

$$
\mu(q):=\sum_{n=0}^{\infty} \frac{(-1)^{n} q^{n^{2}}\left(q ; q^{2}\right)_{n}}{\left(-q^{2} ; q^{2}\right)_{n}^{2}} .
$$

The function $\mu(q)$ appears in Ramanujan's "Lost" Notebook $[9$, Sec 3 with $a=1$ ] 


\section{Hecke type expansion}

We shall use the following result of Bailey to find Hecke type expansion for these mock theta functions: If $\left\{\alpha_{n}\right\}$ and $\left\{\beta_{n}\right\}$ form a Bailey pair relative to $a$, then

(1) $\sum_{n=0}^{\infty} \frac{\left(\rho_{1}\right)_{n}\left(\rho_{2}\right)_{n}\left(a q / \rho_{1} \rho_{2}\right)^{n} \alpha_{n}}{\left(a q / \rho_{1}\right)_{n}\left(a q / \rho_{2}\right)_{n}}$

$$
=\frac{(a q)_{\infty}\left(a q / \rho_{1} \rho_{2}\right)_{\infty}}{\left(a q / \rho_{1}\right)_{\infty}\left(a q / \rho_{2}\right)_{\infty}} \sum_{n=0}^{\infty}\left(\rho_{1}\right)_{n}\left(\rho_{2}\right)_{n}\left(a q / \rho_{1} \rho_{2}\right)^{n} \beta_{n} .
$$

Two sequences $\left\{\alpha_{n}\right\}$ and $\left\{\beta_{n}\right\}, n \geq 0$ form a Bailey pair relative to $a$, if

$$
\beta_{n}=\sum_{r=0}^{n} \frac{\alpha_{r}}{(q)_{n-r}(a q)_{n+r}}
$$

for all $n \geq 0$. Letting $q \rightarrow q^{2}, \rho_{1} \rightarrow \infty$ and taking $\rho_{2}=-q, a=q^{2}$, in (1) we have

$$
\sum_{n=0}^{\infty} \frac{q^{n^{2}+2 n}\left(-q ; q^{2}\right)_{n} \alpha_{n}}{\left(-q^{3} ; q^{2}\right)_{n}}=\frac{\left(q^{4} ; q^{2}\right)_{\infty}}{\left(-q^{3} ; q^{2}\right)_{\infty}} \sum_{n=0}^{\infty} q^{n^{2}+2 n}\left(-q ; q^{2}\right)_{n} \beta_{n} .
$$

If $\alpha_{n}$ and $\beta_{n}$ are Bailey pairs, Andrews[3, (2.13) and (2.14), p.73], then

$$
\begin{aligned}
\alpha_{n}= & \frac{q^{n^{2}}(b c)^{n}\left(1-a q^{2 n}\right)(a / b)_{n}(a / c)_{n}}{(1-a)(q b)_{n}(q c)_{n}} \\
& \times \sum_{j=0}^{n} \frac{(-1)^{j}\left(1-a q^{2 j-1}\right)(a)_{j-1}(b)_{j}(c)_{j}}{q^{\left(\frac{j}{2}\right)}(b c)^{j}(q)_{j}(a / b)_{j}(a / c)_{j}}
\end{aligned}
$$

and

$$
\beta_{n}=\frac{1}{(q b)_{n}(q c)_{n}} .
$$

Letting $q \rightarrow q^{2}$ and taking $a=q^{2}, b=c=q$, in (3) and (4), we have

$$
\alpha_{n}=\frac{(1-q)\left(1+q^{2 n+1}\right)}{(1+q)\left(1-q^{2 n+1}\right)} q^{2 n^{2}+2 n}\left[1+\sum_{j=-n}^{n}(-1)^{j} q^{-\left(j^{2}+j\right)}\right]
$$

and

$$
\beta_{n}=\frac{1}{\left(q^{3} ; q^{2}\right)_{n}^{2}}
$$


Putting these values of $\alpha_{n}$ and $\beta_{n}$ in (2), we have

$$
A(q)=\frac{\bar{J}_{1,2}}{J_{2}} \sum_{n=0}^{\infty} \frac{q^{3 n^{2}+4 n+1}}{\left(1-q^{2 n+1}\right)}\left[1+\sum_{j=-n}^{n}(-1)^{j} q^{-\left(j^{2}+j\right)}\right],
$$

which is the Hecke Type expansion for $A(q)$. have

Letting $q \rightarrow q^{2}, \rho_{1} \rightarrow \infty$ and taking $\rho_{2}=-q^{2}, a=q^{2}$, in (1), we

$$
\sum_{n=0}^{\infty} q^{n^{2}+n} \alpha_{n}=\frac{\left(q^{4} ; q^{2}\right)_{\infty}}{\left(-q^{2} ; q^{2}\right)_{\infty}} \sum_{n=0}^{\infty} q^{n^{2}+n}\left(-q^{2} ; q^{2}\right)_{n} \beta_{n} .
$$

Letting $q \rightarrow q^{2}$ and taking $a=q^{2}, b=c=q$, in (3) and (4), we have

$$
\alpha_{n}=\frac{(1-q)\left(1+q^{2 n+1}\right)}{(1+q)\left(1-q^{2 n+1}\right)} q^{2 n^{2}+2 n}\left[1+\sum_{j=-n}^{n}(-1)^{j} q^{-\left(j^{2}+j\right)}\right]
$$

and

$$
\beta_{n}=\frac{1}{\left(q^{3} ; q^{2}\right)_{n}^{2}} \text {. }
$$

Putting these values of $\alpha_{n}$ and $\beta_{n}$ in (6), we have

$$
B(q)=\frac{\left(-q^{2} ; q^{2}\right)_{\infty}}{\left(q^{2} ; q^{2}\right)_{\infty}} \sum_{n=0}^{\infty} q^{3 n^{2}+3 n} \frac{\left(1+q^{2 n+1}\right)}{\left(1-q^{2 n+1}\right)}\left[1+\sum_{j=-n}^{n}(-1)^{j} q^{-\left(j^{2}+j\right)}\right],
$$

which is the Hecke Type expansion for $B(q)$.

Letting $q \rightarrow q^{2}, \rho_{1} \rightarrow \infty$ and taking $\rho_{2}=q, a=1$, in (1), we have

$$
\sum_{n=0}^{\infty}(-1)^{n} q^{n^{2}} \alpha_{n}=\frac{\left(q^{2} ; q^{2}\right)_{\infty}}{\left(q ; q^{2}\right)_{\infty}} \sum_{n=0}^{\infty}(-1)^{n} q^{n^{2}}\left(q ; q^{2}\right)_{n} \beta_{n}
$$

For the Bailey pairs $\left\{\alpha_{n}\right\}$ and $\left\{\beta_{n}\right\}$, we use the result of Andrews[3. (2.14) and (2.16), pp. 73-74\} for $a=1$

$$
\begin{aligned}
& \alpha_{n}(1, b, c, q) \\
= & \frac{(-1)^{n} q^{\left(\frac{{ }^{n+1}}{2}\right)}\left(1+q^{n}\right)(1-b)(1-c)}{\left(1-q^{n} b\right)\left(1-q^{n} c\right)} \\
& +\frac{q^{n^{2}}(b c)^{n}\left(1-q^{2 n}\right)(1 / b)_{n}(1 / c)_{n}(b c-1)}{(q b)_{n}(q c)_{n}} \\
& \times\left[\frac{1}{(1-b)(1-c)}+\sum_{j=1}^{n-1} \frac{(-1)^{j}\left(1+q^{j}\right)(b)_{j}(c)_{j}}{q^{\left(\frac{j}{2}\right)(b c)^{j+1}(1 / b)_{j+1}(1 / c)_{j+1}}}\right]
\end{aligned}
$$


and

$$
\beta_{n}=\frac{1}{(q b)_{n}(q c)_{n}} .
$$

Letting $q \rightarrow q^{2}$ and taking $b=c=-1$, in (8) and (9), we have

$$
\alpha_{n}=\frac{4(-1)^{n} q^{n^{2}+n}}{\left(1+q^{2 n}\right)}
$$

and

$$
\beta_{n}=\frac{1}{\left(-q^{2} ; q^{2}\right)_{n}^{2}} .
$$

Putting these values of $\alpha_{n}$ and $\beta_{n}$ in (7), we have

$$
\mu(q)=\frac{4\left(q ; q^{2}\right)_{\infty}}{\left(q^{2} ; q^{2}\right)_{\infty}} \sum_{n=0}^{\infty} \frac{q^{2 n^{2}+n}}{1+q^{2 n}}
$$

which is another representation for Ramanujan's function $\mu(q)$.

\section{5. $F_{q}$-functions}

Truesdell[11] calls the functions which satisfy the functional equation

$$
\frac{\partial}{\partial z} F(z, \alpha)=F(z, \alpha+1)
$$

as $F$-functions. The $q$-analogue of $(10)$ is the $q$-differential equation

$$
D_{q, z} F(z, \alpha)=F(z, \alpha+1)
$$

where

$$
z D_{q, z} F(z, \alpha)=F(z, \alpha)-F(z q, \alpha) .
$$

We shall call the functions which satisfy the difference equation (11) as $F_{q}$-functions.

We shall define the following generalized functions

$$
\begin{aligned}
& A(z, \alpha):=\frac{1}{(z)_{\infty}} \sum_{n=0}^{\infty} \frac{(z)_{n} q^{n^{2}+n(\alpha+1)+1}\left(-q ; q^{2}\right)_{n}}{\left(q ; q^{2}\right)_{n+1}^{2}} \\
& B(z, \alpha):=\frac{1}{(z)_{\infty}} \sum_{n=0}^{\infty} \frac{(z)_{n} q^{n^{2}+n \alpha}\left(-q^{2} ; q^{2}\right)_{n}}{\left(q ; q^{2}\right)_{n+1}^{2}} \\
& \mu(z, \alpha):=\frac{1}{(z)_{\infty}} \sum_{n=0}^{\infty} \frac{(-1)^{n}(z)_{n} q^{n^{2}-n+n \alpha}\left(q ; q^{2}\right)_{n}}{\left(-q^{2} ; q^{2}\right)_{n}^{2}}
\end{aligned}
$$


For $z=0, \alpha=1$, they reduce to $A(q), B(q)$ and $\mu(q)$ functions. We shall show that these generalized functions are $F_{q}$-functions. By definition

$$
\begin{aligned}
z D_{q, z} A(z, \alpha)= & A(z, \alpha)-A(z q, \alpha) \\
= & \frac{1}{(z)_{\infty}} \sum_{n=0}^{\infty} \frac{(z)_{n} q^{n^{2}+n(\alpha+1)+1}\left(-q ; q^{2}\right)_{n}}{\left(q ; q^{2}\right)_{n+1}^{2}} \\
& -\frac{1}{(z q)_{\infty}} \sum_{n=0}^{\infty} \frac{(z q)_{n} q^{n^{2}+n(\alpha+1)+1}\left(-q ; q^{2}\right)_{n}}{\left(q ; q^{2}\right)_{n+1}^{2}} \\
= & \frac{1}{(z)_{\infty}} \sum_{n=0}^{\infty} \frac{(z)_{n} q^{n^{2}+n(\alpha+1)+1}\left(-q ; q^{2}\right)_{n}}{\left(q ; q^{2}\right)_{n+1}^{2}} \\
& -\frac{1}{(z)_{\infty}} \sum_{n=0}^{\infty} \frac{(z)_{n} q^{n^{2}+n(\alpha+1)+1}\left(-q ; q^{2}\right)_{n}\left(1-z q^{n}\right)}{\left(q ; q^{2}\right)_{n+1}^{2}} \\
= & \frac{z}{(z)_{\infty}} \sum_{n=0}^{\infty} \frac{(z)_{n} q^{n^{2}+n(\alpha+2)+1}\left(-q ; q^{2}\right)_{n}}{\left(q ; q^{2}\right)_{n+1}^{2}} \\
= & z A(z, \alpha+1) .
\end{aligned}
$$

Hence

$$
D_{q, z} A(z, \alpha)=A(z, \alpha+1) .
$$

So $A(z, \alpha)$ is a $F_{q}$-Function.

A similar proof shows that $B(z, \alpha)$ and $\mu(z, \alpha)$ are $F_{q}$-Functions.

\section{Integral representation}

We now give integral representation for these generalized functions and consequently for the mock theta functions Thomas[5, (1.11.1), p.19] and Jackson[5, (1.11.1), p.19] defined $q$-integral as

$$
\int_{0}^{1} f(t) d_{q} t=(1-q) \sum_{n=0}^{\infty} f\left(q^{n}\right) q^{n} .
$$

The limiting case of the $q$-beta integral $[5,(1.11 .7)$, p.19] is

$$
\frac{1}{\left(q^{x} ; q\right)_{\infty}}=\frac{(1-q)^{-1}}{(q ; q)_{\infty}} \int_{0}^{1} t^{x-1}(t q ; q)_{\infty} d_{q} t .
$$


By definition (12)

$$
A(z, \alpha):=\frac{1}{(z)_{\infty}} \sum_{n=0}^{\infty} \frac{(z)_{n} q^{n^{2}+n(\alpha+1)+1}\left(-q ; q^{2}\right)_{n}}{\left(q ; q^{2}\right)_{n+1}^{2}} .
$$

Writing $q^{z}$ for $z$ and $q^{\alpha}=b$, we have

$$
\begin{aligned}
A\left(q^{z}, \alpha\right) & =\frac{1}{\left(q^{z} ; q\right)_{\infty}} \sum_{n=0}^{\infty} \frac{\left(q^{z} ; q\right)_{n} q^{n^{2}+n(\alpha+1)+1}\left(-q ; q^{2}\right)_{n}}{\left(q ; q^{2}\right)_{n+1}^{2}} \\
& =\sum_{n=0}^{\infty} \frac{q^{n^{2}+n(\alpha+1)+1}\left(-q ; q^{2}\right)_{n}}{\left(q ; q^{2}\right)_{n+1}^{2}\left(q^{n+z} ; q\right)_{\infty}} \\
& =\sum_{n=0}^{\infty} \frac{q^{\left(n^{2}+n+1\right)+n \alpha}\left(-q ; q^{2}\right)_{n}}{\left(q ; q^{2}\right)_{n+1}^{2}} \frac{(1-q)^{-1}}{(q ; q)_{\infty}} \int_{0}^{1} t^{n+z-1}(t q ; q)_{\infty} d_{q} t
\end{aligned}
$$

by (16), with $x$ replaced by $n+z$. Hence

$$
\begin{aligned}
A\left(q^{z}, \alpha\right)= & \frac{(1-q)^{-1}}{(q ; q)_{\infty}} \int_{0}^{1} t^{z-1}(t q ; q)_{\infty} \\
& \times \sum_{n=0}^{\infty} \frac{q^{\left(n^{2}+n+1\right)}\left(-q ; q^{2}\right)_{n}}{\left(q ; q^{2}\right)_{n+1}^{2}}(b t)^{n} d_{q} t
\end{aligned}
$$

But

$$
A(0, \alpha)=\sum_{n=0}^{\infty} \frac{q^{\left(n^{2}+n+1\right)+n \alpha}\left(-q ; q^{2}\right)_{n}}{\left(q ; q^{2}\right)_{n+1}^{2}} .
$$

Since we have put $q^{\alpha}=b$,

$$
A(0, b)=\sum_{n=0}^{\infty} \frac{b^{n} q^{n^{2}+n+1}\left(-q ; q^{2}\right)_{n}}{\left(q ; q^{2}\right)_{n+1}^{2}}
$$

Hence

$$
A(0, b t)=\sum_{n=0}^{\infty} \frac{(b t)^{n} q^{n^{2}+n+1}\left(-q ; q^{2}\right)_{n}}{\left(q ; q^{2}\right)_{n+1}^{2}} .
$$

Replacing the summation on the right-side of (17) by (18), we have

$$
A\left(q^{z}, \alpha\right)=\frac{(1-q)^{-1}}{(q ; q)_{\infty}} \int_{0}^{1} t^{z-1}(t q ; q)_{\infty} A(0, b t) d_{q} t
$$

which is the integral representation for $A\left(q^{z}, \alpha\right)$. Similarly the integral representation for the other two functions

$$
B\left(q^{z}, \alpha\right)=\frac{(1-q)^{-1}}{(q ; q)_{\infty}} \int_{0}^{1} t^{z-1}(t q ; q)_{\infty} B(0, b t) d_{q} t
$$


and

$$
\mu\left(q^{z}, \alpha\right)=\frac{(1-q)^{-1}}{(q ; q)_{\infty}} \int_{0}^{1} t^{z-1}(t q ; q)_{\infty} \mu(0, b t) d_{q} t
$$

\section{Multibasic expansions}

Using the summation formula $[5,(3.6 .7)$, p.71] and $[8$, Lemma 10, p.57], we have the bibasic expansion

$$
\begin{array}{r}
\sum_{k=0}^{\infty} \frac{\left(1-a p^{k} q^{k}\right)\left(1-b p^{k} q^{-k}\right)(a, b ; p)_{k}(c, a / b c ; q)_{k} q^{k}}{(1-a)(1-b)(q, a q / b ; q)_{k}(a p / c, b c p ; p)_{k}} \sum_{m=0}^{\infty} \alpha_{m+k} \\
=\sum_{m=0}^{\infty} \frac{(a p, b p ; p)_{m}(c q, a q / b c ; q)_{m}}{(a p / c, b c p ; p)_{m}(q, a q / b ; q)_{m}} \alpha_{m}
\end{array}
$$

Corollary. Letting $q \rightarrow q^{3}, c \rightarrow \infty$ in (22), we have

$$
\begin{array}{r}
\sum_{k=0}^{\infty} \frac{\left(1-a p^{k} q^{3 k}\right)\left(1-b p^{k} q^{-3 k}\right)(a, b ; p)_{k} q^{\frac{3 k^{2}+3 k}{2}}}{(1-a)(1-b)\left(q^{3}, a q^{3} / b ; q^{3}\right)_{k} b^{k} p^{\frac{k^{2}+k}{2}}} \sum_{m=0}^{\infty} \alpha_{m+k} \\
=\sum_{m=0}^{\infty} \frac{(a p, b p ; p)_{m} q^{\frac{3 m^{2}+3 m}{2}}}{\left(q^{3}, a q^{3} / b ; q^{3}\right)_{m} b^{m} p^{\frac{m^{2}+m}{2}}} \alpha_{m}
\end{array}
$$

We shall use this corollary to give a multibasic expansion of these generalized functions and since for $\alpha=1, x=0$, they reduce to the second order mock theta functions, these multibasic expansion for $\alpha=1$ and $x=0$ will be for the mock theta functions.

(i) Putting $a=\frac{x}{q}, p=q$, and taking

$$
\alpha_{n}=\frac{q^{n \alpha+1}\left(-q ; q^{2}\right)_{n}\left(q^{3} ; q^{3}\right)_{n}\left(x q^{2} / b ; q^{3}\right)_{n} b^{n}}{\left(q ; q^{2}\right)_{n+1}^{2}(b q ; q)_{n}}
$$

in (23), we get after a little simplification,

$$
\begin{aligned}
& (x)_{\infty} A(x, \alpha) \\
& =\sum_{k=0}^{\infty} \frac{\left(1-x q^{4 k-1}\right)\left(1-b q^{-2 k}\right)(x ; q)_{k-1}\left(-q ; q^{2}\right)_{k} q^{k^{2}+k(\alpha+1)+1}}{\left(1-b q^{k}\right)\left(q ; q^{2}\right)_{k+1}^{2}} \\
& \quad \times \phi\left[\begin{array}{l}
q, 0:-q^{2 k+1}, 0: x q^{3 k+2} / b, q^{3 k+3} \\
b q^{k+1}: q^{2 k+3}, q^{2 k+3}: 0,0:
\end{array} ; q, q^{2}, q^{3} ; b q^{\alpha}\right]
\end{aligned}
$$


(ii) Putting $a=\frac{x}{q}, p=q=b$ and taking

$$
\alpha_{n}=\frac{q^{n \alpha}\left(-q^{2} ; q^{2}\right)_{n}\left(q^{3} ; q^{3}\right)_{n}\left(x q ; q^{3}\right)_{n}}{\left(q ; q^{2}\right)_{n+1}^{2}\left(q^{2} ; q\right)_{n}}
$$

in (23), we have

(25) $(x)_{\infty} B(x, \alpha)$

$$
\begin{aligned}
& =\sum_{k=0}^{\infty} \frac{\left(1-x q^{4 k-1}\right)\left(1-q^{-2 k+1}\right)(x ; q)_{k-1}\left(-q^{2} ; q^{2}\right)_{k} q^{k^{2}+k \alpha}}{\left(1-q^{k+1}\right)\left(q ; q^{2}\right)_{k+1}^{2}} \\
& \quad \times \phi\left[\begin{array}{l}
q, 0:-q^{2 k+2}, 0: x q^{3 k+1}, q^{3 k+3}: \\
q^{k+2}: q^{2 k+3}, q^{2 k+3}: 0,0:
\end{array} ; q, q^{2}, q^{3} ; q^{\alpha}\right] .
\end{aligned}
$$

(iii) Putting $a=\frac{x}{q}, p=q, b=q^{2}$ and taking

$$
\alpha_{n}=\frac{(-1)^{n}\left(x ; q^{3}\right)_{n}\left(q^{3} ; q^{3}\right)_{n}\left(q ; q^{2}\right)_{n} q^{n \alpha}}{\left(-q^{2} ; q^{2}\right)_{n}^{2}\left(q^{3} ; q\right)_{n}}
$$

in (23), we have

$$
\text { (26) } \begin{aligned}
& (x)_{\infty} \mu(x, \alpha) \\
= & \sum_{k=0}^{\infty} \frac{(-1)^{k}\left(1-x q^{4 k-1}\right)\left(1-q^{-2 k+2}\right)(x ; q)_{k-1}\left(q ; q^{2}\right) q_{k} q^{k^{2}-k+k \alpha}}{\left(1-q^{k+2}\right)\left(-q^{2} ; q^{2}\right)_{k}^{2}} \\
& \times \phi\left[\begin{array}{l}
q, 0: q^{2 k+1}, 0: x q^{3 k}, q^{3 k+3}: \\
q^{k+3}:-q^{2 k+2},-q^{2 k+2}: 0,0: ; q, q^{2}, q^{3} ;(-q)^{\alpha}
\end{array}\right] .
\end{aligned}
$$

\section{Continued fraction}

McIntosh[7] has shown

$$
\begin{aligned}
& A(q)=\sum_{n=0}^{\infty} \frac{q^{n+1}\left(-q^{2} ; q^{2}\right)_{n}}{\left(q ; q^{2}\right)_{n+1}} \\
& B(q)=\sum_{n=0}^{\infty} \frac{q^{n}\left(-q ; q^{2}\right)_{n}}{\left(q ; q^{2}\right)_{n+1}} .
\end{aligned}
$$

We can write

$$
\begin{aligned}
A(q) & =\frac{q}{1-q} \sum_{n=0}^{\infty} \frac{q^{n}\left(-q^{2} ; q^{2}\right)_{n}\left(q^{2} ; q^{2}\right)_{n}}{\left(q^{2} ; q^{2}\right)_{n}\left(q^{3} ; q^{2}\right)_{n}} \\
& =\frac{q}{1-q} \varphi_{1}\left[\begin{array}{l}
-q^{2}, q^{2} \\
q^{3}
\end{array}, q^{2} ; q\right]
\end{aligned}
$$


and

$$
\begin{aligned}
B(q) & =\frac{1}{1-q} \sum_{n=0}^{\infty} \frac{q^{n}\left(-q ; q^{2}\right)_{n}\left(q^{2} ; q^{2}\right)_{n}}{\left(q^{2} ; q^{2}\right)_{n}\left(q^{3} ; q^{2}\right)_{n}} \\
& =\frac{1}{1-q}{ }^{2} \varphi_{1}\left[\begin{array}{l}
-q, q^{2} \\
q^{3}
\end{array}, q^{2} ; q\right] .
\end{aligned}
$$

We have put $A(q)$ and $B(q)$ as a $2 \varphi_{1}$ series and we have continued fraction representations for ${ }_{2} \varphi_{1}$ series hence $A(q)$ and $B(q)$ can be represented as continued fractions.

We shall show the representation for $A(q)$ as a continued fraction. The representation for $B(q)$ will be on similar lines. Consider the Heine's continued fraction expansion $[2,(9.4)$, p.20]

where

$$
\frac{{ }_{2} \varphi_{1}(a, b ; c ; q, z)}{2 \varphi_{1}(a, b q ; c q ; q, z)}=1-\frac{a_{1} z}{1-} \frac{a_{2} z}{1-} \frac{a_{3} z}{1-\cdots}
$$

and

$$
a_{2 k+1}=\frac{q^{k}\left(1-a q^{k}\right)\left(b-c q^{k}\right)}{\left(1-c q^{2 k}\right)\left(1-c q^{2 k+1}\right)}
$$

$$
a_{2 k}=\frac{q^{k-1}\left(1-b q^{k}\right)\left(a-c q^{k}\right)}{\left(1-c q^{2 k-1}\right)\left(1-c q^{2 k}\right)}
$$

Making $q \rightarrow q^{2}$ and putting $a=q^{2}, b=-q^{2}, c=q^{3}, z=q$ we have

$$
\frac{(1-q)}{q} \frac{A(q)}{{ }_{2} \varphi_{1}\left(q^{2},-q^{4} ; q^{5} ; q^{2}, q\right)}=1-\frac{a_{1} z}{1-} \frac{a_{2} z}{1-} \frac{a_{3} z}{1-\cdots},
$$

where

and

$$
a_{2 k+1}=-\frac{q^{2 k+2}\left(1-q^{2 k+2}\right)\left(1+q^{2 k+1}\right)}{\left(1-q^{4 k+3}\right)\left(1-q^{4 k+5}\right)}
$$

$$
a_{2 k}=\frac{q^{2 k}\left(1+q^{2 k+2}\right)\left(1-q^{2 k+1}\right)}{\left(1-q^{4 k+1}\right)\left(1-q^{4 k+3}\right)} \text {. }
$$

Similarly we can have a continued fraction representation for $B(q)$.

\section{Conclusion}

We have made a very comprehensive study of these mock theta functions. We shall give a modular transformation in a later paper.

ACKNOWLEDGMENTS. I am thankful to the referee for his valuable comments. 


\section{References}

[1] G. E. Andrews, Hecke Modular forms and the Kac-Peterson identities, Trans Amer. Math. Soc. 283 (1984), 451-458.

[2] G. E. Andrews, B. C. Berndt, L. Jacobsen, and R. L. Lamphere, The continued fractions found in the Unorganised portions of Ramanujan's notebooks, Memoir no. 477, American Mathematical Society, Providence, 1992.

[3] G. E. Andrews and D. Hickerson, Ramanujan's "Lost" Notebook VII: The sixth order mock theta functions, Adv. Math. 89 (1991), 60-105.

[4] Youn-Seo Choi, Tenth order mock theta functions in Ramanujan's "Lost" Notebook, Invent. Math. 136 (1999), 497-569.

[5] G. Gasper and M. Rahman, Basic Hypergeometric Series, Cambridge University Press, Cambridge, 1990.

[6] B. Gordon and R. J. McIntosh, Some eight order mock theta functions, J. London Math. Soc. 62 (2000), no. 2, 321-335.

[7] R. J. McIntosh, Second order mock theta function, submitted to Canad. J. Math. 2004.

[8] E. D. Rainville, Special Function, Chelsea Publishing Company, Bronx, New York, 1960.

[9] S. Ramanujan, Collected Paper, Cambridge University Press, London/New York, 1927 (reprinted Chelsea New York, 1962).

[10] Bhaskar Srivastava, An application of the constant term method to Ramanujan's mock theta functions, accepted for publication.

[11] C. Truesdell, An essay toward a unified theory of special functions, Princeton University Press, Princenton, 1948.

Department of Mathematics, LuCknow University, LuCKNow, India

E-mail: bhaskarsrivastav@yahoo.com 